\title{
Adverse effect of excess body weight on survival in cervical cancer patients after surgery and radiotherapy
}

\author{
Yunseon Choi, MD, PhD¹, Ki Jung Ahn, MD, PhD¹, Sung Kwang Park, PhD¹, Heunglae Cho, MD, PhD', \\ Ji Young Lee, $M D^{2}$
}

'Department of Radiation Oncology, ${ }^{2}$ Division of Oncology/Hematology, Department of Internal Medicine, Inje University Busan Paik Hospital, Inje University College of Medicine, Busan, Korea

\begin{abstract}
Purpose: This study aimed to assess the effects of body mass index (BMI) on survival in cervical cancer patients who had undergone surgery and radiotherapy (RT).

Materials and Methods: We retrospectively reviewed the medical records of 70 cervical cancer patients who underwent surgery and RT from 2007 to 2012. Among them, 40 patients (57.1\%) had pelvic lymph node metastases at the time of diagnosis. Sixty-seven patients (95.7\%) had received chemotherapy. All patients had undergone surgery and postoperative RT. Median BMI of patients was $22.8 \mathrm{~kg} / \mathrm{m}^{2}$ (range, 17.7 to $35.9 \mathrm{~kg} / \mathrm{m}^{2}$ ).

Results: The median duration of follow-up was 52.3 months (range, 16 to 107 months). Twenty-four patients (34.3\%) showed recurrence. Local failure, regional lymph nodal failure, and distant failure occurred in 4 (5.7\%), 6 (8.6\%), and 17 (24.3\%) patients, respectively. The 5 -year actuarial pelvic control rate was $83.4 \%$. The 5 -year cancer-specific survival (CSS) and disease-free survival (DFS) rates were $85.1 \%$ and $65.0 \%$, respectively. The presence of pelvic lymph node metastases $(n=30)$ and being overweight or obese $\left(n=34, B M I \geq 23 \mathrm{~kg} / \mathrm{m}^{2}\right)$ were poor prognostic factors for CSS ( $p=0.003$ and $p=0.045$, respectively). Of these, pelvic lymph node metastasis was an independent prognostic factor ( $p=0.030)$ for CSS.

Conclusion: Overweight or obese cervical cancer patients showed poorer survival outcomes than normal weight or underweight patients. Weight control seems to be important in cervical cancer patients to improve clinical outcomes.
\end{abstract}

Keywords: Overweight, Obesity, Survival, Cervical cancer, Radiotherapy

\section{Introduction}

Cervical cancer is one of the most common cancers in women worldwide, even though its mortality rate has declined due to vaccination and active screening programs [1,2]. Generally, stage I-Ila cervical cancer patients are managed through radical hysterectomy. Radiotherapy (RT) without surgery can be used to treat all stages of cervical cancer, with a modest overall survival rate of about $60 \%$ for stage II disease. Although curative RT is the treatment of choice for the International Federation of Gynecology and Obstetrics (FIGO) stage IIb cervical cancer [3], surgical resection is often performed before RT. Compared to RT, surgery is advantageous for younger women who wish to preserve ovarian function. Moreover, surgical resection provides accurate information about the stage of the cancer. After surgical resection, additional

Received 12 September 2016, Revised 1 November 2016, Accepted 9 November 2016.

Correspondence: Yunseon Choi, MD, PhD, Department of Radiation Oncology, Inje University Busan Paik Hospital, Inje University College of Medicine, 75 Bokji-ro, Busanjin-gu, Busan 47392, Korea. Tel: +82-51-890-8606, Fax: +82-51-891-1754, E-mail: rtyoon@gmail.com

(c) This is an Open Access article distributed under the terms of the Creative Commons Attribution Non-Commercial License (http://creativecommons.org/ licenses/by-nc/4.0/) which permits unrestricted non-commercial use, distribution, and reproduction in any medium, provided the original work is properly cited.

www.e-roj.org 
postoperative RT is generally recommended in patients with pelvic lymph node metastases, invasion of paracervical tissue, deep cervical invasion, or positive surgical margins, in order to improve survival outcomes.

More evidence about the relationship between cancer progression and overweight or obesity is available these days [4-6]. According to recent reports, cancer is primarily a metabolic disease [7]. Several clinical studies $[5,6,8]$ have explained the association between obesity and incidence of cancers such as colon cancer, breast cancer, endometrial cancer, cervical cancer, and ovarian cancer. In addition, low levels of adiponectin (an adipocyte-specific protein, which involves insulin resistance) are related to the occurrence of cancers that typically affect women [4].

Occurrence of cervical cancer is related to patient body mass index (BMI) according to recent reports [9-13]. Though the main risk factor for occurrence of cervical cancer is infection by human papillomavirus (HPV) [14], other factors are also involved in the development of cervical cancer. Body weight and calorie intake are shown to influence cervical cancer development. Clark et al. [13] recently reported that extremes of body weight related to reduced survival in cervical cancer patients. Ahn et al. [9] reported that triglyceride levels and impaired fasting glucose levels are prognostic factors for recurrence-free survival in early-stage cervical cancer patients. If being overweight or obese influences cancer treatment outcomes, lifestyle modifications [15] can be important for patients who undergo cancer treatment, not only as primary prevention but also as secondary preventative measure after treatment.

In this study, we evaluated the effect of BMI on the prognosis of Asian cervical cancer patients, after they had undergone surgery and RT. The primary end point of this study was cancer-specific survival (CSS). We hypothesized that obese or overweight cervical cancer patients would have poor treatment outcomes compared to normal or underweight patients.

\section{Materials and Methods}

We retrospectively evaluated the medical records of 70 patients, who were clinically diagnosed with FIGO stage IIb cervical cancer and treated with surgery and RT from March 2007 to September 2012 at Inje University Busan Paik Hospital. This study was approved by the ethics committee of Inje University Busan Paik Hospital. The patients who had received definitive RT without radical surgery were excluded for evaluation. We did not evaluate patients who had abdominal para-aortic or inguinal lymph node metastases at the time of diagnosis, who underwent inappropriate resection (total hysterectomy or trachelectomy) for cancer treatment, who died, or who were lost to follow-up within 12 months of diagnosis.

Obesity and overweight were defined according to Asian $\mathrm{BMI}$ classification. BMI was obtained by dividing body weight $(\mathrm{kg})$ by height $(\mathrm{m})$ squared. Normal weight is defined as a BMI equal or greater than $18.5 \mathrm{~kg} / \mathrm{m}^{2}$ and less than $23 \mathrm{~kg} / \mathrm{m}^{2}$. BMI equal to or greater than $23 \mathrm{~kg} / \mathrm{m}^{2}$ is considered overweight; above $25 \mathrm{~kg} / \mathrm{m}^{2}$, obese; below $18.5 \mathrm{~kg} / \mathrm{m}^{2}$, underweight. Information for the patients' body weight and height was obtained from the electronic anesthesia records on the day of surgery.

All patients received surgical resection as follows; radical abdominal hysterectomy $(n=52)$, radical abdominal hysterectomy with bilateral salpingo-oophorectomy ( $\mathrm{n}=$ 12), and radical abdominal hysterectomy with bilateral pelvic lymphadenectomy $(n=6)$.

Three-dimensional conformal RT was administered to all patients. The daily dose of external beam RT was $1.8 \mathrm{~Gy}$, which was delivered via a four-field box technique to the pelvis for up to 50.4 Gy (range, 45 to $50.4 \mathrm{~Gy}$ ). An additional external beam boost to tumor bed was administered to 3 patients with 3.6-9 Gy. High-dose-rate intracavitary radiation therapy (ICR) was performed in 23 patients (32.9\%), with a pathologically high risk of treatment failure, such as a positive surgical resection margin. The dose of ICR to point A was 10-15 Gy for postoperative RT and was delivered as 3-5 Gy per fraction.

Characteristics of the patients are summarized in Table 1. The median age of the patients was 54.5 years (range, 35 to 77 years). Chemotherapy was administered in 67 patients (95.7\%). The chemotherapy regimen consisted of cisplatin $(n=60)$, 5 -fluorouracil $(n=66)$, or both.

Pelvic failure (in-field failure) was defined as recurrence or progression of cancer at the stump, or pelvic (regional) lymph node failure, or a combination of these characteristics. Pelvic control was calculated from the date of pathologic diagnosis to the date of intra-pelvic failure or of last follow-up. Diseasefree survival (DFS) was calculated from the date of pathologic diagnosis to the date of overall progression (intra-pelvic or distant failure) or of final follow-up. CSS was measured from the date of diagnosis to the date of expiration due to cervical cancer progression or date of final follow-up.

Statistical analysis was performed using SPSS ver. 18.0 (SPSS Inc., Chicago, IL, USA). A p-value of less than 0.05 was 
Table 1. Patient characteristics $(n=70)$

\begin{tabular}{|c|c|}
\hline Characteristic & No. $(\%)$ \\
\hline Age (yr), median (range) & $54.5(35-77)$ \\
\hline \multicolumn{2}{|l|}{ Histology } \\
\hline Squamous cell carcinoma & $62(88.6)$ \\
\hline Adenocarcinoma & $6(8.6)$ \\
\hline Adenosquamous carcinoma & $2(2.9)$ \\
\hline \multicolumn{2}{|l|}{ HPV subtype } \\
\hline 16 and 18 & $29(41.4)$ \\
\hline Others & $12(17.1)$ \\
\hline Not assessed & $29(41.4)$ \\
\hline \multicolumn{2}{|l|}{ Pathologic T stage } \\
\hline T1a-T2a & $43(61.4)$ \\
\hline T2b-Т3a & $27(38.4)$ \\
\hline \multicolumn{2}{|l|}{ Pathologic N stage } \\
\hline NO & $40(57.1)$ \\
\hline $\mathrm{N}_{+}$ & $30(42.9)$ \\
\hline \multicolumn{2}{|l|}{ Resection margin status } \\
\hline Ro & $46(65.7)$ \\
\hline R $1-2$ & $24(34.3)$ \\
\hline \multicolumn{2}{|l|}{ Body mass index $\left(\mathrm{kg} / \mathrm{m}^{2}\right)$} \\
\hline Underweight (<18.5) & $2(2.9)$ \\
\hline Normal weight (18.5-23) & $34(48.6)$ \\
\hline Overweight (23-25) & $17(24.3)$ \\
\hline Obesity $(\geq 25)$ & $17(24.3)$ \\
\hline \multicolumn{2}{|l|}{ Radiotherapy } \\
\hline EBRT only & $47(67.1)$ \\
\hline$E B R T+I C R$ & $23(32.9)$ \\
\hline \multicolumn{2}{|l|}{ Chemotherapy } \\
\hline Yes & 67 (95.7) \\
\hline No & $3(4.3)$ \\
\hline
\end{tabular}

HPV, human papillomavirus; EBRT, external beam radiotherapy; $I C R$, intracavitary radiotherapy.

Table 2. Comparison of patients' clinical factors according to overweight/obesity

\begin{tabular}{lccc}
\hline & $\begin{array}{c}\mathrm{BMI}<23 \\
\left(\mathrm{~kg} / \mathrm{m}^{2}\right)\end{array}$ & $\begin{array}{c}\mathrm{BMI} \geq 23 \\
\left(\mathrm{~kg} / \mathrm{m}^{2}\right)\end{array}$ & p-value \\
\hline $\begin{array}{l}\text { Local failure } \\
\text { Yes }\end{array}$ & 2 & 2 & 0.671 \\
$\quad$ No & 34 & 32 & \\
Regional lymph nodal failure & & & 0.087 \\
$\quad$ Yes & 1 & 5 & \\
$\quad$ No & 35 & 29 & \\
$\begin{array}{l}\text { Distant failure } \\
\text { Yes }\end{array}$ & & & 0.554 \\
$\quad$ No & 9 & 8 & \\
Overall progression & 27 & 26 & \\
Yes & & & 0.177 \\
No & 10 & 14 & \\
\hline
\end{tabular}

BMI, body mass index. considered statistically significant. The chi-square test was used to compare two groups to find the clinical factors related to BMI and to estimate the effects of excess body weight on treatment failure. The Kaplan-Meier method was used to estimate pelvic control, DFS, and CSS. Rates of survival and pelvic control were compared using a log-rank test. The Cox regression hazard model was used to assess independent prognostic factors for survival.

\section{Results}

\section{Pattern of failure}

The median duration of follow-up was 52.3 months (range, 16 to 107 months). Overall, 24 patients experienced recurrence. Ten patients (14.3\%) showed intra-pelvic recurrence (in-field failure, including 4 local failures and 6 pelvic lymph nodal failures) and 17 patients (21.4\%) experienced distant failure. Three patients showed both intra-pelvic recurrence and distant failure during the follow-up period. The most frequent distant failure sites were the lungs $(n=3)$. Nine patients $(12.9 \%)$ died during the follow-up period, because of disease progression.

Table 2 shows pattern of failure correlated with BMI values (BMI $<23 \mathrm{~kg} / \mathrm{m}^{2}$ or BMI $\geq 23 \mathrm{~kg} / \mathrm{m}^{2}$ ). Local failure (operation site or marginal failure), pelvic failure (in-field failure, including local failure and regional lymph nodal failure), and overall progression were not significantly different between the overweight or obese patients (BMI $\geq 23 \mathrm{~kg} / \mathrm{m}^{2}, \mathrm{n}=34$ ) and the normal weight or underweight patients $(p=0.224, p=$ 0.224 , and $p=0.124$, respectively). However, regional lymph nodal failure tended to be higher in the overweight or obese patients than in normal or underweight patients ( $p=0.087)$.

\section{Actuarial pelvic control}

The 5 -year actuarial pelvic control rate was $83.4 \%$. Fig. 1 shows the pelvic control, DFS, and CSS. Underweight or normal weight patients did not have a better actuarial pelvic control rate than overweight or obese patients $(p=0.123,5$-year pelvic control, $\mathrm{BMI}<23 \mathrm{~kg} / \mathrm{m}^{2}$ [91.5\%] vs. BMI $\geq 23 \mathrm{~kg} / \mathrm{m}^{2}$ [73.2\%]).

\section{Survival analysis}

The 5-year CSS and DFS rates were $85.1 \%$ and $65.0 \%$, respectively (Fig. 1). In further analysis, the 5 -year DFS rate was not differentiated by obesity or overweight $(p=0.224)$.

Table 3 shows the prognostic factors for CSS. Overall, overweight/obesity (Fig. 2) and pelvic lymph node involvement (Fig. 3) influenced CSS ( $p=0.045$ and $p=0.003$, respectively) 
in the univariate analysis. Obesity $(n=17)$ alone also tended to influence the CSS $(p=0.141)$. There was no significant effect of ICR $(n=23)$ boost on CSS ( $p=0.522)$. High risk

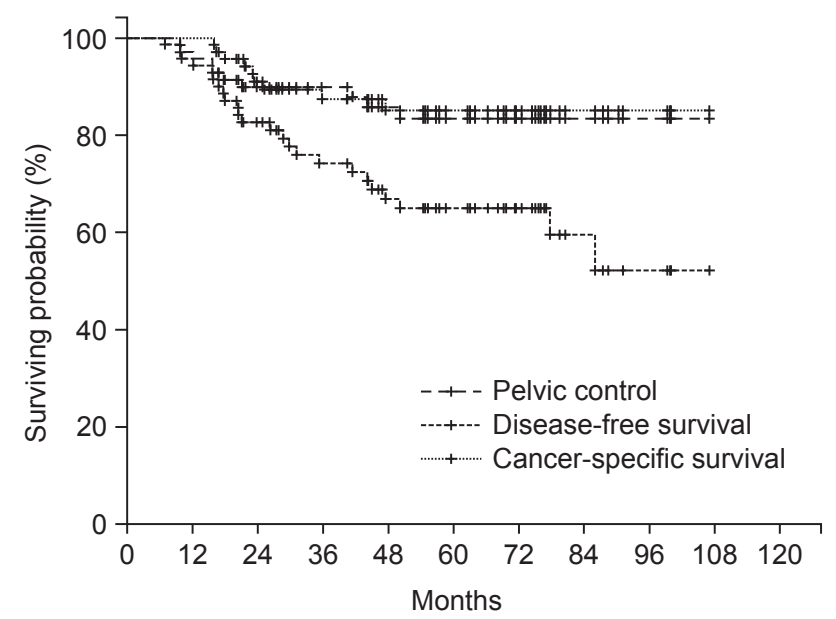

Fig. 1. Pelvic control, disease-free survival, and cancer-specific survival.
HPV subtypes (16 and 18) did not affect the CSS ( $p=0.868$ ). Multivariate analysis (Table 3 ) showed that pelvic lymph node metastasis was an independent prognostic factor for CSS ( $p=$ 0.03).

\section{Discussion and Conclusion}

The results of our study show the detrimental effects of overweight or obesity on survival in cervical cancer patients treated with surgery and RT. Survival was related to BMI in cervical cancer. This result reflects the nature of cervical cancer as a metabolic disease.

There are some possible explanations for why BMI can influence the outcome of cervical cancer. Although HPV is the main cause of cervical cancer, PI3K/Akt pathway, has an important role in cancer development $[16,17]$. Cervical cancer frequently showed activated PI3K pathway. Moreover, PI3K-Akt-mTOR signals amplified by HPV oncogenes E5/E6/ E7 activation [18]. This PI3K-Akt-mTOR pathway is important not only in cancer progression but also in cell metabolism.

Table 3. Prognostic factors for cancer-specific survival (CSS)

\begin{tabular}{|c|c|c|c|c|c|}
\hline & \multirow{2}{*}{ No. } & \multicolumn{2}{|c|}{ Univariate analysis } & \multicolumn{2}{|c|}{ Multivariate analysis } \\
\hline & & $5-y r$ CSS & $p$-value & $p$-value & $\mathrm{HR}(95 \% \mathrm{Cl})$ \\
\hline Age $(y r)$ & & & 0.836 & - & - \\
\hline$<55$ & 35 & 83 & & & \\
\hline$\geq 55$ & 35 & 87.8 & & & \\
\hline Histology & & & 0.195 & - & - \\
\hline Squamous cell carcinoma & 62 & 87.3 & & & \\
\hline Adenocarcinoma/adenosquamous carcinoma & 8 & 57.1 & & & \\
\hline Pathologic T stage & & & 0.146 & - & - \\
\hline T1a-T2a & 43 & 89.6 & & & \\
\hline T2b-T3a & 27 & 77.3 & & & \\
\hline Pathologic N stage & & & 0.003 & 0.030 & $10.1(1.3-81.7)$ \\
\hline No & 40 & 95.8 & & & \\
\hline $\mathrm{N}+$ & 30 & 71.6 & & & \\
\hline Resection margin status & & & 0.560 & & \\
\hline Ro & 46 & 86.8 & & & \\
\hline $\mathrm{R} 1-2$ & 24 & 82 & & & \\
\hline Body mass index $\left(\mathrm{kg} / \mathrm{m}^{2}\right)$ & & & 0.045 & 0.138 & $3.3(0.7-16.1)$ \\
\hline Underweight/normal weight $(<23)$ & 36 & 93.5 & & & \\
\hline Overweight/obesity ( $\geq 23$ ) & 34 & 75.7 & & & \\
\hline Radiotherapy & & & 0.522 & - & - \\
\hline EBRT only & 47 & 87.5 & & & \\
\hline$E B R T+I C R$ & 23 & 81 & & & \\
\hline Chemotherapy & & & 0.147 & - & - \\
\hline Yes & 67 & 86.3 & & & \\
\hline No & 3 & 50 & & & \\
\hline
\end{tabular}

$\mathrm{HR}$, hazard ratio; $\mathrm{Cl}$, confidence interval; EBRT, external beam radiotherapy; ICR, intracavitary radiotherapy. 


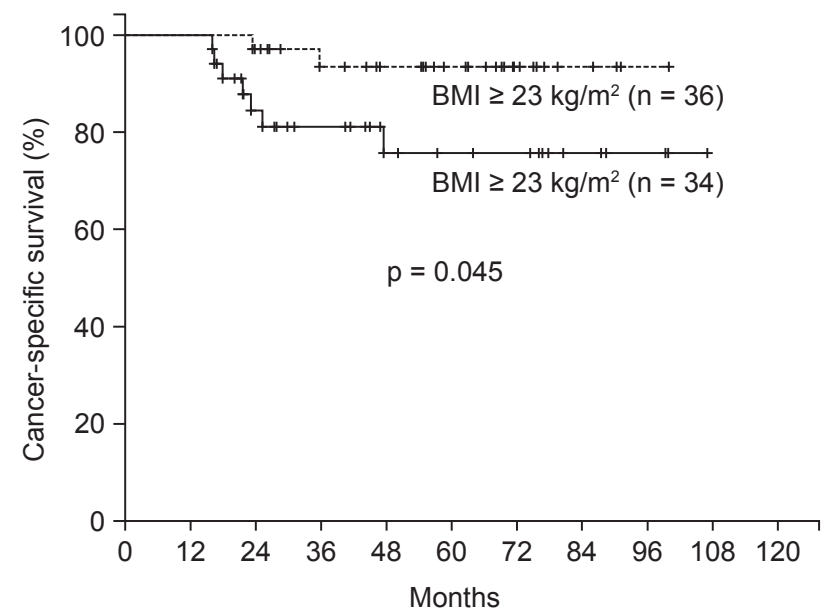

Fig. 2. Cancer-specific survival by overweight or obesity.

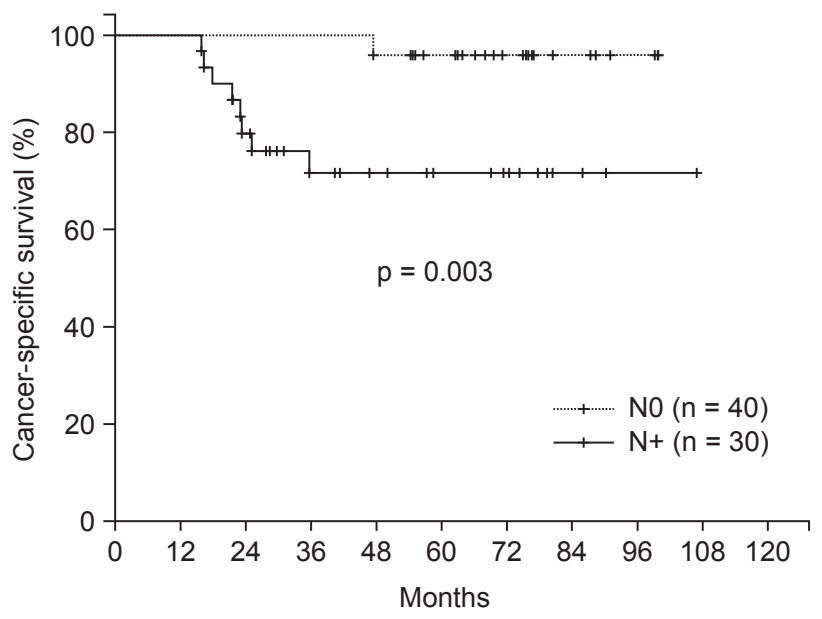

Fig. 3. Cancer-specific survival by pelvic lymph node metastases.

According to Yuan et al. [19], Leptin can increase the proliferation of cervical cancer cells. They argued that Leptin can be involved in the progression of cervical cancer by activation of c-myc and bcl-2 pathway. Also, Leptin receptor stimulation could activate the PI3K-Akt pathway. Moreover, Statins are known to have anti-inflammatory and vascular endothelial protection properties after RT [20]. In other words, lipid lowering drugs can improve the RT outcomes.

During cancer diagnosis, obese patients could have a lower detection rate in Pap smear tests than normal weight patients, because of the difficulty in proper sampling [21]. During treatment, the rates of complete resection or extent of lymphadenectomy in overweight or obese patients might be lower than those in normal weight patients, because of the difficulty of surgical approach [22]. Furthermore, body volume could affect the radiation dose-distribution during RT. The possibility of set-up uncertainty might increase in overweight or obese patients.

Several studies which validate the detrimental effects of overweight or obesity in cervical cancer patients have been reported $[10,13,23]$. A recent meta-analysis showed that higher BMI was clinically related with cervical cancer risk [10]. Clark et al. [13] also showed that excess body mass index (BMI $\geq 25$ $\mathrm{kg} / \mathrm{m}^{2}$ ) was associated with worse survival in cervical cancer patients.

Our study also showed that the importance of body weight in prognosis existed in cervical cancer patients who underwent surgery and RT. Though the standard of overweight/obesity is different to Western standard, BMI can be an important prognostic factor for Asian cervical cancer patients. In this study, the cut-off value of BMI that determined survival was $23 \mathrm{~kg} / \mathrm{m}^{2}$ (overweight). The standard of BMl for overweight, according to the World Health Organization (WHO) criteria, is lower in Korea than in Western countries ( $\geq 23 \mathrm{~kg} / \mathrm{m}^{2}$ vs. $\geq 25$ $\mathrm{kg} / \mathrm{m}^{2}$ ).

Further evaluation is needed to delineate the precise mechanism of the detrimental effects of overweight or obesity on the prognosis of cervical cancer. The pattern of failure might be different in overweight or obese patients than in normal weight patients. In this study, the rate of regional lymph node failure in overweight or obese patients was slightly higher than in normal weight patients, although there was no statistical difference between the two values. Overweight or obese patients might be more easily exposed to regional lymph nodal failures.

There is a need to find effective strategies for early detection or prevention of cervical cancer relapses that are related to excess body weight in patients. The prognostic effects of physical activity and lifestyle change should be more evaluated in the next level studies.

In this study, regional lymph nodal recurrence rate tended to be higher in patients with overweight/obesity. Moreover, pelvic lymph node metastases were related to survival rates after treatment. Therefore, intensity-modulated RT can be used for pelvic lymph nodal irradiation, in order to enable appropriate radiation dose escalation, as this is closely related to increased local control. In addition, more attention should be paid to overweight and obese cancer patients, to find evidence of cancer recurrence in the follow-up periods after treatment. For early detection of recurrence, reducing the follow-up period for overweight or obese patients can be a good strategy.

Limitations of this study should be considered. The number 
of patients studied was limited to only 70 and this study was based on data from a single institution. Especially, the number of obesity patients was only 17. Thus, it was difficult to get statistically relevant results in this analysis. Moreover, this study was confined to patients who received surgery and RT. Therefore, the association between BMI and cancer progression in patients who underwent RT without surgery was difficult to estimate based on the current study results. More studies are needed to identify the clinical effects of weight reduction in overweight or obese cancer patients, specifically with respect to cancer progression and survival. The discrepancy of detrimental effects of overweight/obesity in treatment failure and survival outcomes should be more evaluated.

In conclusion, overweight or obese cervical cancer patients showed poor survival outcome compared to normal weight or underweight patients. The importance of body weight in the prognosis of cervical cancer after treatment should not be ignored. Careful observation is needed for overweight or obese cervical cancer patients. Maintaining normal body weight or underweight seems to improve cervical cancer survival outcome. Recommendation of lifestyle modifications can be considered an important aspect of clinical management for cervical cancer patients who have undergone surgery and RT.

\section{Conflict of Interest}

No potential conflict of interest relevant to this article was reported.

\section{References}

1. Ferlay J, Soerjomataram I, Dikshit R, et al. Cancer incidence and mortality worldwide: sources, methods and major patterns in GLOBOCAN 2012. Int J Cancer 2015;136:E359-86.

2. Lee JY, Kim EY, Jung KW, et al. Trends in gynecologic cancer mortality in East Asian regions. J Gynecol Oncol 2014;25:17482.

3. Colombo N, Carinelli S, Colombo A, et al. Cervical cancer: ESMO Clinical Practice Guidelines for diagnosis, treatment and follow-up. Ann Oncol 2012;23 Suppl 7:vii27-32.

4. Nagaraju GP, Rajitha B, Aliya $S$, et al. The role of adiponectin in obesity-associated female-specific carcinogenesis. Cytokine Growth Factor Rev 2016;31:37-48.

5. Jee SH, Yun JE, Park EJ, et al. Body mass index and cancer risk in Korean men and women. Int J Cancer 2008;123:1892-6.

6. Renehan AG, Zwahlen M, Egger M. Adiposity and cancer risk: new mechanistic insights from epidemiology. Nat Rev Cancer 2015;15:484-98.
7. Seyfried TN, Flores RE, Poff AM, D'Agostino DP. Cancer as a metabolic disease: implications for novel therapeutics. Carcinogenesis 2014;35:515-27.

8. Keum N, Greenwood DC, Lee DH, et al. Adult weight gain and adiposity-related cancers: a dose-response meta-analysis of prospective observational studies. J Natl Cancer Inst 2015;107:djv088.

9. Ahn HK, Shin JW, Ahn HY, et al. Metabolic components and recurrence in early-stage cervical cancer. Tumour Biol 2015;36:2201-7.

10. Poorolajal J, Jenabi E. The association between BMI and cervical cancer risk: a meta-analysis. Eur J Cancer Prev 2016;25:232-8.

11. Frumovitz $M$, Jhingran $A$, Soliman PT, Klopp AH, Schmeler KM, Eifel PJ. Morbid obesity as an independent risk factor for disease-specific mortality in women with cervical cancer. Obstet Gynecol 2014;124:1098-104.

12. Lopez-Hernandez D. Epidemiological association between body fat percentage and cervical cancer: a cross-sectional population-based survey from Mexico. Arch Med Res 2013:44:454-8.

13. Clark $L H$, Jackson $A L$, Soo $A E$, Orrey DC, Gehrig PA, Kim KH. Extremes in body mass index affect overall survival in women with cervical cancer. Gynecol Oncol 2016;141:497-500.

14. Dona MG, Vocaturo A, Giuliani M, et al. p16/Ki-67 dual staining in cervico-vaginal cytology: correlation with histology, Human Papillomavirus detection and genotyping in women undergoing colposcopy. Gynecol Oncol 2012;126:198202.

15. Schlumbrecht MP, Sun CC, Huang MS, Zandstra F, Bodurka DC Lifestyle modification in cervical cancer survivors: an ongoing need. Int J Gynecol Cancer 2014;24:570-5.

16. Lou $H$, Villagran $G$, Boland JF, et al. Genome analysis of Latin American cervical cancer: frequent activation of the PIK3CA pathway. Clin Cancer Res 2015;21:5360-70.

17. Bregar AJ, Growdon WB. Emerging strategies for targeting PI3K in gynecologic cancer. Gynecol Oncol 2016;140:333-44.

18. Zhang L, Wu J, Ling MT, Zhao L, Zhao KN. The role of the PI3K/ Akt/mTOR signalling pathway in human cancers induced by infection with human papillomaviruses. Mol Cancer 2015;14:87.

19. Yuan Y, Zhang J, Cai L, et al. Leptin induces cell proliferation and reduces cell apoptosis by activating c-myc in cervical cancer. Oncol Rep 2013;29:2291-6.

20. Kim JH, Jenrow KA, Brown SL. Mechanisms of radiationinduced normal tissue toxicity and implications for future clinical trials. Radiat Oncol J 2014;32:103-15.

21. Maruthur NM, Bolen SD, Brancati FL, Clark JM. The association of obesity and cervical cancer screening: a systematic review and meta-analysis. Obesity (Silver Spring) 2009;17:375-81.

22. Papadia A, Ragni N, Salom EM. The impact of obesity on 
surgery in gynecological oncology: a review. Int J Gynecol Cancer 2006;16:944-52.

23. Lee JK, So KA, Piyathilake CJ, Kim MK. Mild obesity, physical activity, calorie intake, and the risks of cervical intraepithelial neoplasia and cervical cancer. PLoS One 2013;8:e66555. 